ABSTRACT: Electrophysiological testing remains an important efficacy parameter in clinical neuropathy trials. The quality of nerve conduction studies in reported trials varies greatly, and may be responsible for negative results. We report the utilization of an expert core lab for electrophysiological testing. With the core lab, the variability of repeat testing is comparable to that of a single, excellent laboratory. Motor conduction velocities demonstrated a coefficient of variation of $3 \%$ and sensory conduction velocities $4 \%$ across 60 study sites. The distal motor evoked potential amplitudes varied by $13 \%$ at the ankle, and $10 \%$ at the wrist. The sensory potential amplitudes varied by $16 \%$ at the ankle, and $11 \%$ at the wrist in 60 sites. The overall monitoring rate in all submitted nerve conduction tracings was $36.6 \%$. Our results show that an expert core lab can improve the electrophysiological quality of clinical trial data with the potential to show small changes in nerve conduction velocities and in both motor and sensory potential amplitudes.

() 1998 John Wiley \& Sons, Inc. Muscle Nerve 21: 1368-1373, 1998

\title{
ELECTROPHYSIOLOGICAL MONITORING IN CLINICAL TRIALS
}

\author{
VERA BRIL, MD, FRCP(C), ${ }^{1}$ RUSSELL ELLISON, MD ${ }^{2}$ MYLAN NGO, RT, ${ }^{1}$ \\ BENGT BERGSTROM, MD, ${ }^{2}$ DONNA RAYNARD, RT, ${ }^{1}$ HUGO GIN, ${ }^{1}$ and the \\ Roche Neuropathy Study Group \\ ${ }^{1}$ University of Toronto, The Toronto Hospital, General Division, EN11-209, 200 \\ Elizabeth Street, Toronto, Ontario, M5G 2C4, Canada \\ ${ }^{2}$ Hoffmann-LaRoche, Toronto, Canada \\ Accepted 16 February 1998
}

Electrophysiological testing (nerve conduction studies, NCS) remains an essential technique for quantifying peripheral nerve function in peripheral neuropathy trials due to its inherent reliability, reproducibility, and objectivity. ${ }^{4,6-9}$ Nerve conduction studies measure peripheral nerve function directly without subjective bias and without contamination by central nervous system pathways. In research trials, neuropathy is defined currently on the basis of two out of five possible areas of abnormality: symptoms, signs, NCS, quantitative sensory testing (QST), and autonomic tests. ${ }^{2}$ The least variable and most reliable is the NCS. ${ }^{10}$ In addition, regulatory bodies have stated the requirement of more than one parameter showing positive change in order to accept a compound's efficacy. Clinical measures of symptoms and signs as used routinely are highly variable.

\footnotetext{
Abbreviations: EMG, electromyographic; HLR, Hoffmann-LaRoche; NCS, nerve conduction studies; QST, quantitative sensory testing; SOM, standard operating manual

Key words: nerve conduction studies; electrophysiology; diabetic neuropathy; clinical trials; monitoring laboratory

Correspondence to: Dr. Vera Bril

CCC 0148-639X/98/111368-06

(C) 1998 John Wiley \& Sons, Inc.
}

Quantitative clinical scales have been developed, but are cumbersome, and not widely employed as they too are variable, particularly for symptoms. ${ }^{7}$ QST has drawbacks as to reliability, reproducibility, and site of pathology. ${ }^{11,12}$

In early studies, uncontrolled nerve conduction evaluations were undertaken. Those responsible for analyses had little appreciation of the technical limitations of electrophysiology. A high degree of variability in parameters was observed: a much greater degree than repeat studies in a single laboratory. ${ }^{3,10}$ Consequently, the power of these studies to detect any meaningful change was severely limited. ${ }^{10} \mathrm{An}$ advance in NCS was the institution of a central expert monitoring facility to provide quality control of the nerve conduction studies. The expert reviewer improved the variability of repeat measures and the power of studies. This function was still limited in its ability to evaluate data, and ensure rigorous protocol adherence and technical competence.

We report a method of optimizing electrophysiological testing in large-scale, multicenter trials. The reproducibility of NCS achieved in 60 sites is that observed in a single, excellent laboratory when strict 
adherence to study protocols is carried out, and when an expert core lab is used to monitor all the electrophysiological data.

\section{MATERIALS AND METHODS}

As part of a large-scale diabetic polyneuropathy trial involving 60 centers in Europe and North America, a core lab was set up at The Toronto Hospital by an experienced electromyographer and neurologist (VB) to evaluate electrophysiological tracings obtained on patients in this study. The core lab developed the protocol for electrophysiological testing to be used by all centers. The core lab reviewed the qualifications of all electrophysiologists and technicians at each study site. In order to participate in this trial, the electrophysiologist was required to be a full member of the American Association of Electrodiagnostic Medicine, or have equivalent training and credentials. All patients had screening nerve conduction studies, then triplicate measurements at the beginning of the study and after 12 months. The patients all had a standard battery of nerve conduction studies including: peroneal motor, bilateral surals, median motor, and sensory. The curves were faxed or couriered to the core lab, where they were entered into a database. A 1-cm scale was attached to each tracing to judge any distortion in faxed tracings. If the signal was distorted, the tracings were then couriered to the core lab. All identifying information as to site, date of testing, investigator, and patient name was eliminated, and a random number assigned to the curve. The information was recorded in a Filemaker Pro database for the Macintosh system. The curves were assessed in a blinded fashion by an electromyography (EMG) technician, then by a second EMG technician, as to adherence to protocol (side tested, baseline-to-peak amplitudes, onset latencies, temperature control, averaging of sensory potentials) and quality of tracing (flatness of baseline, motor contamination of sensory potentials, similarity of motor curves with different sites of stimulation along the same nerve, and accuracy of calculations). After this review and acceptance of the tracings, the case report forms were completed by the core lab. If the curves failed to meet protocol requirements, corrections were made on the curve (e.g., correcting latency measurement from the peak to curve onset, or amplitude from peak-to-peak to baseline-to-peak), the site was asked for any missing information (e.g., side tested, distance, temperature), or the site was asked to repeat the tracing in cases of excessive artifact. After review and acceptance, all curves for 1 patient for 1 day's nerve conduction studies were reassembled, and reviewed for a final time. At this point, some additional aberrations were discovered: e.g., identical curves submitted for two different days, wrong side tested on a nerve, a leg changing length by $20 \mathrm{~cm}$ in 1 day. The site would be asked to provide correct data, or repeat the tracing. The core lab sent reports to each site on each set of nerve conduction tracings, and the data were sent to a double-data-entry facility. The completed case report forms were sent to each site.

Prior to randomizing patients in the study, each site completed 4 normal subjects twice to familiarize the sites with the protocol, and the procedures. Training meetings were held with groups of investigators to review in a minute fashion the protocol and standard operating procedures. Each site was provided with a standard operating manual (SOM) of the nerve conduction and reporting procedures. Each site was monitored by Hoffmann-LaRoche (HLR) staff, and if necessary, the core lab performed site audits.

Sites were standardized with respect to background and training of personnel prior to starting the study. The SOM outlined all core lab procedures, NCS procedures, and gave examples of poor waveforms.

Testing was standardized as to temperature, side of testing, stimulation protocol, averaging sensory potentials, marking latencies and amplitudes, and providing information for the core lab. Repeat testing could be done on consecutive days, but not sooner. Prior to randomization of patients to active treatment, all nerve conduction studies had to be approved and accepted in the placebo run-in baseline. All reviews were blinded. To maintain quality assurance, an external laboratory reviewed a subsample of the tracings, and provided opinions as to the actions taken by the core lab.

Compliance of the sites with the core lab activities was integral to the study and participation in the trial as mandated by HLR and supported for the duration of the trial. All sites agreed to these procedures prior to starting the study.

The core lab maintained a detailed standard operating procedure on site, and underwent auditing by HLR personnel twice during the trial to ensure strict compliance with protocol procedures.

We examined the mean values and variability of repeat testing for all study centers, and then for geographic areas (Canada, Europe, and the U.S.). Statistical analyses were done using Statview 4 for the Macintosh. We computed means \pm standard errors for normative subjects, means \pm standard deviations for patients, and coefficients of variation as a percentage for repeat testing. 


\section{RESULTS}

Sixty sites participated in the trial. Compliance with core lab functions was ensured by the sponsor of the program (HLR), who was committed to the core lab concept. Most sites accepted the concept readily, although full compliance from every site required extensive discussion and education as the value of consistency and standardization across all sites.

The core lab screened data from 1474 patients recruited at 60 sites. Thirteen sites were in Canada (495 patients), 16 in Europe (285 patients), and 31 were in the U.S. (565 patients). Of these patients, 1345 were randomized and continued with the trial and 1144 , or $85 \%$, completed the trial.

NCS in 253 normal subjects were done twice in 1-3 months. The recruitment interval extended over 9 months. The study interval was 12 months. Fiftyseven normal subjects had repeat testing at the end of the double-blind interval. Turnaround time of the blinded review required 6 working days due to the blinding of the review process and the high volume of nerve conduction studies which arrived in the core lab daily at the peaks of recruitment and completion (up to 50 sets, or 350 individual nerves). Our methodology was unique in that the review process was completely blinded, and the curves were reread, and measurements redone, if needed, to comply with protocol requirements. In essence, all data were centralized.
Patients were selected for mild neuropathy as shown by the values of the nerve conduction parameters in the control subjects and patients with diabetic polyneuropathy (Table 1). Many values fall within the lower range of normal. In addition, no significant change in conduction velocities or amplitudes were observed over a 12-month study interval, in either placebo- or drug-treated patients in this trial. This indicates that at this early stage of neuropathy, very little, if any, deterioration in electrophysiology occurs in 12 months.

The variability of repeat testing is shown in Table 2 , with a breakdown for different nerves. At baseline, the variability was $13 \%$ for peroneal motor amplitude at the ankle, and $15 \%$ at the knee. For median motor, the amplitude variability was $10 \%$ at the wrist and $11 \%$ at the elbow. The numbers were slightly lower at the completion of the study. The motor nerve conduction velocities varied by only $3 \%$ in arm and leg across 60 sites at baseline and completion.

More striking is the variability of sensory studies. Sural conduction velocities varied by $5 \%$ at the onset, and $4 \%$ at the study termination. Median nerve sensory conduction varied by $4 \%$. The sural sensory amplitudes varied by $16 \%$ at onset and $15 \%$ at the termination, and median sensory amplitudes varied by $11 \%$ at the wrist and $17 \%$ at the elbow throughout the study.

The Canadian sites had the lowest variability in

Table 1. Sixty-center trial nerve conduction data.

\begin{tabular}{|c|c|c|c|}
\hline \multirow[b]{2}{*}{ Parameter } & \multirow[b]{2}{*}{ Control subjects } & \multicolumn{2}{|c|}{ Patients } \\
\hline & & Baseline & Completion \\
\hline$n$ & 253 & 1345 & 1144 \\
\hline Median motor DL & $3.3 \pm 0.03$ & $4.0 \pm 0.9$ & $4.1 \pm 0.8$ \\
\hline Median motor amp wrist & $10.4 \pm 0.2$ & $8.2 \pm 2.7$ & $8.4 \pm 2.7$ \\
\hline Median motor amp elbow & $9.7 \pm 0.2$ & $7.5 \pm 2.7$ & $7.8 \pm 2.6$ \\
\hline Median motor CV & $58.5 \pm 0.3$ & $51.2 \pm 4.7$ & $51.0 \pm 4.5$ \\
\hline Median sensory DL & $2.5 \pm 0.02$ & $3.1 \pm 0.6$ & $3.1 \pm 0.7$ \\
\hline Median sensory amp wrist & $37.5 \pm 1.05$ & $15.4 \pm 9.4$ & $15.3 \pm 9.3$ \\
\hline Median sensory distal CV & $58.1 \pm 0.4$ & $47.6 \pm 8.0$ & $47.4 \pm 7.9$ \\
\hline Median sensory amp elbow & $17.5 \pm 0.6$ & $7.1 \pm 4.3$ & $7.0 \pm 4.3$ \\
\hline Median sensory proximal CV & $62.9 \pm 0.3$ & $55.0 \pm 5.0$ & $54.8 \pm 4.8$ \\
\hline Peroneal motor DL & $4.3 \pm 0.04$ & $4.9 \pm 1.1$ & $4.9 \pm 1.0$ \\
\hline Peroneal motor amp ankle & $6.2 \pm 0.1$ & $4.0 \pm 2.2$ & $4.0 \pm 2.3$ \\
\hline Peroneal motor amp knee & $5.7 \pm 0.1$ & $3.5 \pm 2.0$ & $3.5 \pm 2.1$ \\
\hline Peroneal motor CV & $48.9 \pm 0.2$ & $40.4 \pm 5.0$ & $40.2 \pm 5.1$ \\
\hline Right sural DL & $2.9 \pm 0.02$ & $3.3 \pm 0.6$ & $3.3 \pm 0.5$ \\
\hline Right sural amp & $14.8 \pm 0.4$ & $5.5 \pm 3.8$ & $5.5 \pm 3.9$ \\
\hline Right sural CV & $49.0 \pm 0.3$ & $41.7 \pm 5.9$ & $42.0 \pm 5.8$ \\
\hline Left sural DL & $2.9 \pm 0.02$ & $3.3 \pm 0.6$ & $3.3 \pm 0.5$ \\
\hline Left sural amp & $15.1 \pm 0.5$ & $5.6 \pm 3.9$ & $5.5 \pm 4.0$ \\
\hline Left sural CV & $48.8 \pm 0.3$ & $41.8 \pm 5.9$ & $41.8 \pm 5.9$ \\
\hline
\end{tabular}

$D L$, distal latency (ms); amp, amplitude (motor, $m V$; sensory, $\mu \mathrm{V}$ ); $\mathrm{CV}$, conduction velocity ( $\mathrm{m} / \mathrm{s}$ ). Values for control subjects are expressed as arithmetic means \pm standard error; values for patients are expressed as arithmetic means \pm standard deviation. 


\begin{tabular}{lccr}
\hline \multicolumn{3}{c}{ Table 2. Variability of repeat testing. } \\
\hline & Control & \multicolumn{2}{c}{ Patients } \\
\cline { 2 - 4 } Purameter & 253 & 1345 & 1144 \\
\hline$n$ & 4 & 4 & 4 \\
Median motor DL & 7 & 10 & 9 \\
Median motor amp wrist & 8 & 11 & 10 \\
Median motor amp elbow & 3 & 3 & 3 \\
Median motor CV & 4 & 4 & 4 \\
Median sensory DL & 8 & 11 & 11 \\
Median sensory amp wrist & 3 & 4 & 4 \\
Median sensory distal CV & 13 & 17 & 17 \\
Median sensory amp elbow & 3 & 4 & 3 \\
Median sensory proximal CV & 5 & 6 & 6 \\
Peroneal motor DL & 9 & 13 & 12 \\
Peroneal motor amp ankle & 10 & 15 & 13 \\
Peroneal motor amp knee & 3 & 3 & 3 \\
Peroneal motor CV & 5 & 6 & 6 \\
Right sural DL & 10 & 16 & 15 \\
Right sural amp & 3 & 5 & 4 \\
Right sural CV & 4 & 6 & 5 \\
Left sural DL & 10 & 16 & 15 \\
Left sural amp & 3 & 5 & 4 \\
Left sural CV & & & \\
\hline
\end{tabular}

$D L$, distal latency (ms); amp, amplitude (motor, $m V$; sensory, $\mu V$ ); $C V$, conduction velocity $(\mathrm{m} / \mathrm{s})$. Values are expressed as percentages.

the trial, as shown in Table 3. For motor amplitudes the variability in Canadian patients was $11 \%$ at onset, compared to $9 \%$ at termination for distal peroneal, and $8 \%$ and $7 \%$ for distal median. The motor conduction velocities were $3 \%$ for both. Sural nerve conduction velocities varied by $4 \%$ in Canada throughout the trial, and the amplitudes by $13 \%$ at onset and by $11 \%$ and $10 \%$ at termination. Similar values were found for median nerve sensory activity.
The rejection and query rate was lowest for Canada with an absolute rejection rate of $4 \%$ at baseline, compared to $11 \%$ for both Europe and the U.S. The overall rejection rate was $8.7 \%$ at baseline. The correction rate was $22.6 \%$ for Canada, $38.4 \%$ for Europe, and $31.4 \%$ for the U.S. An overall rate of $29.6 \%$ was found at entry of patients. In summary, the core lab had comments, corrections, or rejections on fully $38.2 \%$ of all nerve conduction study tracings submitted to the core lab. All sites improved at completion of the trial. The rejection rates were lower: overall $6.3 \%$ for the study, $2.8 \%$ for Canada, 9.6\% for Europe, and $7.9 \%$ for the U.S. The correction rate was $27.9 \%$ overall, $14.1 \%$ in Canada, $41.4 \%$ in Europe, and $32.8 \%$ in the U.S. Overall, the core lab had comments, corrections, or rejections on $34.3 \%$ at completion. The overall number of tracings with some change in the trial was $36.6 \%$, or 3300 of 9021 individual nerve tracings.

Concordance with the external monitoring facility was extremely high at about $95 \%$ agreement on actions between the two facilities. Disagreements most often concerned review of isolated tracings by the external laboratory, and were resolved when all repetitions of the same nerve conduction study were available for assessment.

\section{DIscussion}

Electrophysiological testing is an efficacy parameter in widespread use and is the most reliable quantitative method currently available to study nerve function in polyneuropathy. The method is limited by the variability of repeat testing, which is large in re-

\begin{tabular}{|c|c|c|c|c|c|c|c|c|}
\hline \multirow[b]{2}{*}{ Parameter } & \multicolumn{4}{|c|}{ Baseline } & \multicolumn{4}{|c|}{ Completion } \\
\hline & All & CAN & EUR & USA & All & CAN & EUR & USA \\
\hline$n$ & 1345 & 285 & 565 & 1144 & 442 & 239 & 463 & 495 \\
\hline Median motor amp wrist & 10 & 8 & 12 & 11 & 9 & 7 & 11 & 10 \\
\hline Median motor amp elbow & 11 & 8 & 13 & 13 & 10 & 7 & 12 & 11 \\
\hline Median motor CV & 3 & 3 & 4 & 4 & 3 & 3 & 3 & 3 \\
\hline Median sensory amp wrist & 11 & 10 & 13 & 12 & 11 & 10 & 11 & 12 \\
\hline Median sensory distal CV & 4 & 4 & 4 & 4 & 4 & 3 & 4 & 4 \\
\hline Median sensory amp elbow & 17 & 15 & 18 & 19 & 17 & 15 & 18 & 19 \\
\hline Median sensory proximal CV & 4 & 4 & 4 & 4 & 3 & 3 & 4 & 4 \\
\hline Peroneal motor amp ankle & 13 & 11 & 15 & 15 & 12 & 9 & 14 & 14 \\
\hline Peroneal motor amp knee & 15 & 12 & 16 & 16 & 13 & 11 & 15 & 15 \\
\hline Peroneal motor CV & 3 & 3 & 4 & 4 & 3 & 3 & 4 & 4 \\
\hline Right sural amp & 16 & 13 & 18 & 17 & 15 & 11 & 17 & 17 \\
\hline Right sural CV & 5 & 4 & 5 & 5 & 4 & 4 & 4 & 5 \\
\hline Left sural amp & 16 & 13 & 19 & 17 & 15 & 10 & 17 & 16 \\
\hline Left sural CV & 5 & 4 & 5 & 5 & 4 & 4 & 4 & 5 \\
\hline
\end{tabular}

All, total patient population; CAN, Canadian patients; EUR, European patients; USA, American patients; Amp, amplitude; CV, conduction velocity. Values are expressed as percentages. 
ported multicenter trials. ${ }^{4,13}$ For this reason, conduction velocity alone has been the major end point, and amplitude measures have not been used in the past, although they are being used now. ${ }^{1}$ With our methods of using a core lab for quality control, of generating data centrally, of maintaining adherence to protocol and excellence of technique, we achieved a degree of variability in nerve conduction studies in 60 centers previously observed only in single excellent laboratories. ${ }^{5}$ This low variability was universal: motor and sensory nerve conduction velocities and amplitudes in upper and lower limbs. Motor nerve conduction velocities showed the least variability $(3 \%)$. The sensory nerve conduction velocities varied between 3 and 5\%. Amplitudes had greater variability, with upper limb motor amplitudes varying up to $10 \%$, and those in the leg up to $15 \%$. Sensory potential amplitudes in the leg had up to $16 \%$ variability, and the arm distally $11 \%$, but $17 \%$ at the elbow. These values across 60 sites are the same as observed in a single excellent laboratory. ${ }^{5}$ The requirement for blinding is not absolute, but does eliminate any possibility of bias in the review process. Unblinded review would require much less time in the core lab, as paper handling would be greatly reduced, although bias in monitoring could be claimed.

Despite efforts by all centers to follow protocol requirements in the manner dictated by this study, our overall $36.6 \%$ rate of intervention indicates the absolute need for an experienced, insightful, and knowledgeable core lab, and for sponsor commitment to the core lab principle.

Variability in 60 sites can be the same as that of a single excellent laboratory using these methods. Consequently, the number of patients required to show small changes (as expected in nerve conduction velocity) is not as great, and the ability to show some change in amplitude is present. Studies with fewer patients would be powered to show these results. This study had the power to show a change of $0.2 \mathrm{~m} / \mathrm{s}$ in conduction velocity between treatment groups and a $0.4-\mu \mathrm{V}$ change in amplitude, neither of which occurred.

We believe this level of variability can be achieved in any multicenter trial, and that this methodology sets a new standard for electrophysiological testing in clinical trials in any polyneuropathy.

We noted some regional differences in variability of testing and query rates, as shown in Tables 3 and 4 , but overall the 60 sites showed excellent compliance with protocol and study procedures, although the rejection/query rates remained above $30 \%$ for the entire trial, confirming the utility of a core lab.

Finally, the lack of change in conduction velocity or amplitude over 12 months is in concordance with the results of the Statil trial done in a similarly affected population of patients for 18 months. ${ }^{13}$ This suggests that the decline in nerve conduction velocity and amplitude in diabetic polyneuropathy are not necessarily a linear function of time in early neuropathy, and that these parameters may be static during this phase of diabetic polyneuropathy. This finding has implications for trials in which halting of definite deterioration is required to show the efficacy of a treatment. It may be that a more advanced population needs to be studied if decline in NCS in 12 months is essential to show efficacy, since other studies have shown decline in more affected individuals at entry. The pattern of decline over years is uncertain, as few studies have followed NCS in patients annually from diagnosis, and our study was not a natural history trial. Limiting trials to mildly affected patients may limit any electrophysiological efficacy that can be demonstrated in 12 months. If only mildly affected individuals are likely to benefit from a therapy, then a requirement for lengthy 5-10-year trials may be present.

In summary, we present the value of core monitoring laboratories for electrophysiological monitoring in clinical trials. Utilization of such laboratories increases the power of studies which use nerve conduction studies as an efficacy parameter, allows, fewer patients to be studied, and provides the poten-

\begin{tabular}{|c|c|c|c|c|c|c|c|c|}
\hline \multirow[b]{2}{*}{ Parameter } & \multicolumn{4}{|c|}{ Baseline } & \multicolumn{4}{|c|}{ Completion } \\
\hline & All & CAN & EUR & USA & All & CAN & EUR & USA \\
\hline Visits accepted & 61.8 & 73.3 & 50.3 & 57.4 & 65.7 & 83.1 & 48.8 & $\overline{59.3}$ \\
\hline Visits accepted with correction & 29.6 & 22.6 & 38.4 & 31.4 & 27.9 & 14.1 & 41.4 & 32.8 \\
\hline Visits rejected & 8.7 & 4.1 & 11.2 & 11.2 & 6.3 & 2.8 & 9.6 & 7.9 \\
\hline Monitoring rate & 38.2 & 26.7 & 49.7 & 42.6 & 34.3 & 16.9 & 51.0 & 40.7 \\
\hline
\end{tabular}

Visit $=$ one complete set of 6 or 7 nerve conduction tracings. The number of interventions per set of tracings is not listed, and varied from 1 to 10. All, total patient population; CAN, Canadian patients; EUR, European patients; USA, American patients. Values are expressed as percentages. Monitoring rate $=\%$ of tracings requiring intervention. 
tial to detect small differences in amplitude and conduction velocity. Our results set a new standard in electrophysiological testing in multicenter trials.

\section{THE ROCHE NEUROPATHY STUDY GROUP}

The Roche Neuropathy Study Group comprises 60 study sites. The listing shows principal investigator, electromyographer/neurophysiologist (if different from principal investigator), city, and country in alphabetical order: A. Belanger, L. Cote, Montreal, Canada, T. Benstead, L.P. Heffernan, Halifax, Canada, R. Bergenstal, F. Taylor, Minneapolis, USA, R. Bernstein, R. Miller, San Francisco, USA, F.W. Bertelsmann, R.L.M. Strijers, Amsterdam, The Netherlands, A. Boulton, W. Schady, A. Carrington, Manchester, U.K., V. Bril, Toronto, Canada, D. Brunet, Quebec City, Canada, W. Carter, J. Nickols, S. Rudnicki, Little Rock, USA, A. Charles, A. Starr, Y. Zhu, Irvine, USA, D. Clarke, D. Smith, Salt Lake City, USA, V. Cwik, Edmonton, Canada, S. DeCherney, R. Fisher, Newark, USA, S. Dippe, R. Goodell, Scottsdale, USA, J. Dupre, I. Hramiak, London, Canada, C.J.V. Fox, A. Bissessar, Northampton, U.K., R. Freeman, Boston, USA, C. Godin, A. Lamontagne, Sherbrooke, Canada, R. Goldberg, K. Sharma, M. Kato, Miami, USA, D.A. Greene, E. Feldman, Ann Arbor, USA, G. Grunberger, J.F. Selwa, Detroit, USA, Y. Harati, C. Gooch, R. Kolimas, Houston, USA, K. Hermansen, J. Christensen, V. Nielson, Aarhus, Denmark, J. Hilsted, M. Damholt, L.B. Blatt, Hvidovre, Denmark, I. Ipp, T. Anderson, Torrence, USA, P. Jennings, C.K. Laljee, York, U.K., J. Jervell, E. Jorum, T. Ganes, Oslo, Norway, F. Kennedy, W. Litchy, Rochester, MN, USA, C. Kilo, R. Frere, B. Green, St. Louis, USA, G. King, J. Rosenszweig, A. Herzog, Boston, USA, V. Koivisto, A.M. Seppalainen, Helsinki, Finland, D. Lau, P. Bourque, D. Preston, Ottawa, Canada, S. Levin, S.A. Chrissian, Los Angeles, USA, A. MacCuish, P. Jamal, Glasgow, U.K., J.I. Malone, J. Korthals, Tampa, USA, L. Olansky, W.D. Shipley, Oklahoma City, USA, M. Pfeifer, J. Farquhar, Springfield, IL, USA, N. Pillay, Winnipeg, Canada, D. Porte, Seattle, USA, G. Poticha, S. Gulevich, Denver, USA, P. Raskin, R. Greenlee, Dallas, USA, G. Rayman, S.J. Wroe, Suffolk, U.K., J. Rosenstock, F. Gul, Dallas, USA, C. Saudek, D. Cornblath, Baltimore, USA, J. Scarpello, P. Heath, Staffordshire, U.K., A. Scheen,
J.M. Crielaard, F.C. Wang, Liege, Belgium, J. Schoelmerich, B. Zietz, Regensberg, Germany, A. Schuaib, P. Siemens, Saskatoon, Canada, P. Sheehan, T. Herron, Westlake, USA, J. Skyler, C. Vandenakker, Miami, USA, D. Studney, Vancouver, Canada, M. Swenson, San Diego, USA, M. Uusitupa, J. Partanen, E. Mervaala, Kuopio, Finland, A. Vinik, M. Holland, Norfolk, USA, J. Wales, I. Smith, Leeds, U.K., D. Zieglar, R. Piolot, Dusseldorf, Germany, D. Zochodne, Calgary, Canada.

Professor J. Arezzo is acknowledged for unstinting advice and encouragement during the trial and in the manuscript preparation. Contract grant sponsor: Hoffmann-LaRoche.

\section{REFERENCES}

1. Arezzo JC: The use of electrophysiology for the assessment of diabetic neuropathy. Neurosci Res Com 1997;21:13-23.

2. Asbury AK, Porte D Jr.: Report and recommendations of the San Antonio Conference on Diabetic Neuropathy. Ann Neurol 1988;24:99-104.

3. Bril V: Role of electrophysiological studies in diabetic neuropathy. Can J Neurol Sci 1994;21:S8-S12.

4. Chaudhry V, Corse A, Freimer M, Glass J, Mellits E, Kuncl R, Quaskey S, Cornblath D: Inter- and intraexaminer reliability of nerve conduction measurements in patients with diabetic neuropathy. Neurology 1994;44:1459-1462.

5. Daube J: Electrophysiologic testing in diabetic neuropathy. Diabet Neuropathy 1987;1:162-176.

6. Dyck P, Karnes J, O'Brien P, et al.: The Rochester Diabetic Neuropathy Study: reassessment of tests and criteria for diagnosis and staged severity. Neurology 1992;42:1164-1170.

7. Dyck P, Kratz K, Lehman K, et al.: The Rochester Diabetic Neuropathy Study: design, criteria for types of neuropathy, selection bias, and reproducibility of neuropathic tests. Neurology 1991;41:799-807.

8. Honet J, Jebsen R, Perrin E: Variability of nerve conduction velocity determinations in normal persons. Arch Phys Med Rehabil 1968;00:650-654.

9. Lamontagne A, Buchthal F: Electrophysiological studies in diabetic neuropathy. J Neurol Neurosurg Psychiatry 1970;33: $442-452$.

10. Macleod A, Till S, Sonksen P: Discussion of the clinical trials of the aldose reductase inhibitor, tolrestat. Int Proc J 1991;4: $17-24$.

11. Maser R, Nielsen V, Bass E, Manjoo Q, Dorman J, Kelsey S, Becker D, Orchard T: Measuring diabetic neuropathy: assessment and comparison of clinical examination and quantitative sensory testing. Diabetes Care 1989;12:270-275.

12. Proceedings of a consensus development conference on standardized measures in diabetic neuropathy. Neurology 1992;42: 1823-1829.

13. Sundkvist G, Armstrong F, Bradbury J, Chaplin C, Ellis S, Owens D, Rosen I, Sonksen P, Trial UKSP: Peripheral and autonomic nerve function in 259 diabetic patients with peripheral neuropathy treated with ponalrestat (an aldose reductase inhibitor) or placebo for 18 months. J Diabetes Complications 1992;6:123-130. 